\title{
Adaptive Data-based Predictive Control for Short Take-off and Landing (STOL) Aircraft
}

\author{
Jonathan S. Barlow* \\ Stinger-Gaffarian Technologies, NASA Ames Research Center, Moffett Field, California, 94035 \\ Diana M. Acosta ${ }^{\dagger}$ \\ NASA Ames Research Center, Moffett Field, California, 94035 \\ and \\ Minh Q. Phan ${ }^{\ddagger}$ \\ Dartmouth College, Hanover, New Hampshire, 03755
}

\begin{abstract}
Data-based Predictive Control is an emerging control method that stems from Model Predictive Control (MPC). MPC computes current control action based on a prediction of the system output a number of time steps into the future and is generally derived from a known model of the system. Data-based predictive control has the advantage of deriving predictive models and controller gains from inputoutput data. Thus, a controller can be designed from the outputs of complex simulation code or a physical system where no explicit model exists. If the output data happens to be corrupted by periodic disturbances, the designed controller will also have the built-in ability to reject these disturbances without the need to know them. When data-based predictive control is implemented online, it becomes a version of adaptive control. The characteristics of adaptive data-based predictive control are particularly appropriate for the control of nonlinear and time-varying systems, such as Short Take-off and Landing (STOL) aircraft. STOL is a capability of interest to NASA because conceptual Cruise Efficient Short Take-off and Landing (CESTOL) transport aircraft offer the ability to reduce congestion in the terminal area by utilizing existing shorter runways at airports, as well as to lower community noise by flying steep approach and climb-out patterns that reduce the noise footprint of the aircraft. In this study, adaptive data-based predictive control is implemented as an integrated flight-propulsion controller for the outer-loop control of a CESTOL-type aircraft. Results show that the controller successfully tracks velocity while attempting to maintain a constant flight path angle, using longitudinal command, thrust and flap setting as the control inputs.
\end{abstract}

\section{Introduction}

Data-based Predictive Control is an emerging control method that stems from Model Predictive Control (MPC). MPC is the concept where the current control action is based on a prediction of the system output a number of time steps into the future. ${ }^{1,2,3}$ A sequence of control actions (from the present time to some future time) is computed that minimizes a finite-duration cost function. Out of this sequence, only the present control input is applied to the system. At the next time step, the entire process repeats. Thus the starting and ending time steps of the cost function shift one time step forward. The term "receding-horizon" is often associated with this strategy.

\footnotetext{
* Research Engineer, Stinger-Gaffarian Technologies, NASA Ames Research Center M/S 269-1, Moffett Field, California, 94035, Member

${ }^{\dagger}$ Research Engineer, NASA Ames Research Center M/S 269-1, Moffett Field, California, 94035, Member

${ }^{\ddagger}$ Professor, Dartmouth College, Hanover, New Hampshire, 03755
} 
In MPC, the system output is generally predicted using the state-space model based approach or the input-output model approach. These two approaches can be unified via an interaction matrix, which offers a convenient mapping from the state-space description to the input-output description. ${ }^{4,5}$ One important feature of the interaction matrix formulation is that although the starting point of the MPC derivation is state-space based, the controller in the end has dynamic output feedback form and can be implemented without an observer.

Using the interaction matrix formulation, MPC combines well with system identification to produce socalled "data-based" designs. Data-based designs are advantageous because they can be designed from the outputs of complex simulation code or a physical system where no explicit model exists. Also, if the output data happens to be corrupted by periodic disturbances, then the designed controller will have the built-in ability to reject these disturbances without the need to know them. When data-based predictive control is implemented online, it becomes a version of adaptive control.

While data-based predictive control has been successfully employed for a number of applications, including linear time-invariant systems and multiple-vehicle formations, ${ }^{6,7}$ the characteristics of data-based predictive control are particularly appropriate for the control of nonlinear and time-varying systems, such as Short Take-off and Landing (STOL) aircraft. STOL is a key capability for future aircraft. Conceptual Cruise Efficient Short Take-off and Landing (CESTOL) transport aircraft offers the ability to reduce congestion in the terminal area by utilizing existing shorter runways at airports and flying approach and climb-out patterns that do not interfere with current air traffic. The CESTOL transport aircraft also offers the ability to lower community noise by flying steep approach and climb-out patterns that reduce the noise footprint of the aircraft. These qualities correspond to the objectives of NASA's vision for the Next Generation Air Transportation System. Consequently, the CESTOL aircraft is one of the Next Generation aircraft configurations targeted by the NASA Aeronautics Subsonic Fixed Wing Project.

In this study, a data-based predictive controller is developed for the outer-loop integrated flightpropulsion control of a CESTOL-type aircraft. The paper begins with an overview of data-based predictive control, followed by a description of the application to the control of the CESTOL-type aircraft. Simulation results and a performance analysis are presented for the vehicle and control system.

\section{Data-based Predictive Control Approach}

\section{A. State-space and Input-output Representations}

Consider an $r$-input, $m$-output system with the system state $x(k)$ and output $y(k)$ given by

$$
\begin{aligned}
& x(k+1)=A x(k)+B u(k)+B_{d} u_{d}(k) \\
& y(k)=C x(k)+D u(k)
\end{aligned}
$$

We assume that neither the system model, defined by $A, B, B_{d}, C$, and $D$, nor the initial state of the system, $x(0)$, are known, but a set of sufficiently rich and long excitation input $u(k)$ and possibly disturbance-corrupted output data $y(k)$ is available. The disturbance input, $u_{d}(k)$, if present, is assumed to be a sum of a finite number of unknown harmonics. Only an upper bound of the number of harmonics is known.

The representation of the data history can be simplified by the introduction of "super-vector" notation, defined by

$$
g_{w}(k) \equiv\left[\begin{array}{c}
g(k) \\
g(k+1) \\
\vdots \\
g(k+w-1)
\end{array}\right]
$$

where $g$ will generally represent an output or control input (column) vector, and $\mathrm{w}$ is the length of the vector. 
For the system in Eq. (1), the output $\mathrm{y}(k)$ is dependent on the initial state $x(0)$ and the disturbance inputs $u_{d}(k)$. Since the disturbance input and the initial state are assumed unknown, it is beneficial to describe the system using a relationship between the excitation input and disturbance-corrupted output that does not explicitly include the terms involving the initial state and the disturbances. In Ref. 4, the interaction matrix formulation captures this input-output relationship, which does not depend on initial state and disturbances. It was shown that the following relationship holds for excitation input and possibly disturbance-corrupted output,

$$
y_{s}(k+q)=\mathbf{P}_{1} u_{p}(k-p)-\mathbf{P}_{2} y_{p}(k-p)+\mathbf{W} u_{s+q}(k)
$$

when $p$ is selected such that $m p \geq n+2 f+1$ and $0 \leq q \leq p$, where $n$ is the system order, $f$ is the number of distinct disturbance frequencies, and the 1 accounts for a constant disturbance if present. In this context, $\mathrm{p}$ is the number of past data points, $\mathrm{q}$ is the start of the prediction horizon, and $\mathrm{s}$ is the length of the prediction horizon. A conservative value for $p$ can be chosen using an upper bound on the order of the system and the number of distinct disturbance frequencies.

\section{B. Model-Predictive Controller Design}

A predictive controller can be designed to minimize the receding-horizon cost function

$$
J(k)=\left(\begin{array}{l}
{\left[y_{s}(k+q)-z_{s}(k+q)\right]^{T} Q\left[y_{s}(k+q)-z_{s}(k+q)\right]} \\
+u_{s+q}^{T}(k) R u_{s+q}(k) \\
+\left[u_{s+q}(k)-u_{s+q}(k-1)\right]^{T} R_{S}\left[u_{s+q}(k)-u_{s+q}(k-1)\right]
\end{array}\right),
$$

where $z_{s}(k+q)$ is the desired output trajectory to be tracked. The output error cost is evaluated over the interval from time $k+q$ to $k+s+q-1$, with a weight matrix of $Q$. The control input cost is evaluated over the interval from time $k$ to $k+s+q-1$, with a weight matrix of $R$. The control smoothing cost, which is the weighted norm of the difference between the control input at time $k$ and the previous control input at time $k-1$, is evaluated over the interval from time $k$ to $k+s+q-1$, with weighting matrix $R_{S}$.

The future control input history $u_{s+q}(k)$ that minimizes the resultant cost function can be found by combining Eq. (3) with Eq. (4), and taking the derivative with respect to $u_{s+q}(k)$, where $u_{s+q}(k)$ becomes

$$
u_{s+q}(k)=A_{1} u_{p}(k-p)+A_{2} y_{p}(k-p)+M_{1} z_{s}(k+q)+M_{2} u_{k-1}
$$

where $\quad A_{1}=-\Gamma^{-1} \mathbf{W}^{T} Q P_{1}, \quad A_{2}=\Gamma^{-1} \mathbf{W}^{T} Q P_{2}, \quad M_{1}=\Gamma^{-1} \mathbf{W}^{T} Q, \quad M_{2}=\Gamma^{-1}\left(\mathrm{I}-L_{S}\right)^{T} R_{S}$, $\Gamma=\left(R+\left(\mathrm{I}-L_{S}\right)^{T} R_{S}\left(\mathrm{I}-L_{S}\right)+\mathbf{W}^{T} Q \mathbf{W}\right), L_{S} \equiv\left[\begin{array}{cccc}0 & 0 & 0 & 0 \\ \mathrm{I}_{r \times r} & 0 & 0 & 0 \\ 0 & \ddots & 0 & 0 \\ 0 & 0 & \mathrm{I}_{r \times r} & 0\end{array}\right]$, and $u_{k-1}=\left[\begin{array}{c}u(k-1) \\ 0_{(s+q-1) r \times 1}\end{array}\right]$.

The optimal control law is extracted from the first $r$ rows of Eq. (5), resulting in the dynamic output feedback form shown in Eq. (6).

$$
u(k)=G u_{p}(k-p)+H y_{p}(k-p)+K z_{s}(k+q)+V u_{k-1}
$$

The gains $\mathrm{G}, \mathrm{H}$, and $\mathrm{K}$, are the first $r$ rows of $\mathbf{A}_{1}, \mathbf{A}_{2}$, and $M_{1}$, respectively. $\mathrm{V}$ is the first $r$ rows of $M_{2}$, which can also be shown to be 


$$
V=\left(I_{r \times(s+q) r}-S\right) \beta
$$

where $\beta=\left[R+\left(\mathrm{I}-L_{S}\right)^{T} R_{S}\left(\mathrm{I}-L_{S}\right)\right]^{-1}\left(\mathrm{I}-L_{S}\right)^{T} R_{S} \quad$ and $\quad S$ is the first $r$ rows of $\left\{I-\Gamma^{-1}\left[R+\left(\mathrm{I}-L_{S}\right)^{T} R_{S}\left(\mathrm{I}-L_{S}\right)\right]\right\}$.

\section{Data-based and Adaptive Controller design}

To employ the optimal control law in Eq. (6), the controller gains $G, H, K$, and $V$ must be either known a priori or estimated online. The controller gains can be designed a priori for the model predictive approach by relying on a model of the system. Similarly, a data-based design can be accomplished a priori in a two step approach, where the model $\mathrm{P}_{1}, \mathrm{P}_{2}, \mathrm{~W}$, is identified from input-output data and the gains $G$, $H, K$, and $V$ are then designed. For an online, adaptive implementation of the control laws, the gains are designed directly from input-output data via a relationship that relates $G, H$, and $K$ to input-output data. The equation that enables the direct relationship is shown in Eq. (8).

$$
\left\{I-\Gamma^{-1}\left[R+\left(\mathrm{I}-L_{S}\right)^{T} R_{S}\left(\mathrm{I}-L_{S}\right)\right]\right\} u_{s+q}(k)=\mathbf{A}_{1} u_{p}(k-p)+\mathbf{A}_{2} y_{p}(k-p)+M_{1} y_{s}(k+q)
$$

Further detail about the derivation of this equation can be found in Ref. 8.

The first $r$ rows of Eq. (8) are extracted to produce the input-output relationship shown in Eq. (9).

$$
S u_{s+q}(k)=G u_{p}(k-p)+H y_{p}(k-p)+K y_{s}(k+q)
$$

As stated previously, $S$ is the first $r$ rows of $\left\{I-\Gamma^{-1}\left[R+\left(\mathrm{I}-L_{S}\right)^{T} R_{S}\left(\mathrm{I}-L_{S}\right)\right]\right\}$. Eq. (9) has the property of being an open-loop input-output equation with the controller gains $G, H$, and $K$ from Eq. (6) included explicitly as coefficients of the equation. Using Eq. (9), the coefficients of the open-loop inputoutput model can be identified, and used in Eq. (6) as the gains of a dynamic feedback controller.

The data-based predictive controller developed for this study updates $G, H$, and $K$ online using Eq. (9) and past input and output data. Since Eq. (9) is a non-causal input-output relationship, the approach begins with a time shift of $-(s+q)$ to the data sets within Eq. (9) in order to fully populate the supervectors of collected data, with the most recent data used being $y(k-1)$ and $u(k-1)$. The time-shifted equation is then

$$
S u_{s+q}(k-s-q)=G u_{p}(k-p-s-q)+H y_{p}(k-p-s-q)+K y_{s}(k-s)
$$

Eq. (10) is then arranged in the form

$$
S u_{s+q}(k-s-q)=\gamma(k) \Gamma(k-1)
$$

where

$$
\gamma(k)=\left[\begin{array}{lll}
G(k) & H(k) & K(k)
\end{array}\right] \text { and } \Gamma(k-1)=\left[\begin{array}{c}
u_{p}(k-p-s-q) \\
y_{p}(k-p-s-q) \\
y_{s}(k-s)
\end{array}\right]
$$

In general, any linear estimation algorithm may be use to identify the parameters in $\gamma$. For this application a recursive least-squares ${ }^{9}$ estimation of the form 


$$
\begin{gathered}
\gamma(k)=\gamma(k-1)+\left\{S u_{s+q}(k-q-s)-\gamma(k-1) \Gamma(k-1)\right\} \frac{\Gamma(k-1)^{T} \Theta(k-1)^{T}}{1+\Gamma(k-1)^{T} \Theta(k-1) \Gamma(k-1)} \\
\Theta(k)=\Theta(k-1)-\frac{\Theta(k-1) \Gamma(k-1) \Gamma(k-1)^{T} \Theta(k-1)^{T}}{1-\Gamma(k-1)^{T} \Theta(k-1) \Gamma(k-1)}
\end{gathered}
$$

is used to update $G, H$, and $K$ in $\gamma$, starting with some initially large covariance matrix $\Theta(0)$ and an initial guess of the controller gains $\gamma(0)$ and $S$. In practice, the control error $\left\{S u_{s+q}(k-q-s)-\gamma(k-1) \Gamma(k-1)\right\}$ found in Eq. (13) is subject to a dead-band. The estimation of $\gamma$ is conducted every time step. The optimal control input can be found as

$$
u(k)=G(k) u_{p}(k-p)+H(k) y_{p}(k-p)+K(k) z_{s}(k+q)+V u_{k-1}
$$

for a set of desired outputs $z_{s}(k+q)$.

\section{Application of Data-based Predictive Control for the Varying Flight Dynamics of STOL}

The data-based predictive control approach is applied to a CESTOL-type aircraft in this study. As mentioned in the Introduction, the data-based predictive control characteristics are beneficial for the control of STOL operation.

STOL operation is unique in that the flight dynamics vary significantly, and that the variation incorporates the aerodynamic and propulsion response of the aircraft. Unlike conventional transport aircraft, which can be controlled with one technique by consistently flying on the frontside of the airspeed versus power required curve (or power curve), a CESTOL transport aircraft will transition between the frontside and backside of the power curve for take-off and landing, and be therefore subject to a transition in control effectiveness. The transition from frontside to backside can be illustrated using gamma-V diagrams. A gamma-V diagram is a contour plot of constant pitch and constant thrust lines as functions of flight path angle (gamma) and velocity (V). On the frontside of the power curve, a change in engine power (thrust) will mainly affect the velocity and an adjustment in aircraft attitude (pitch angle) will mainly affect the flight path. ${ }^{10}$ These relationships can be seen in Fig. 1 as horizontal pitch lines and somewhat vertical thrust lines. The opposite is true on the backside of the power curve - a change in engine power will mainly affect the flight path and an adjustment in aircraft attitude will mainly affect the velocity. ${ }^{10}$ These relationships can be seen in Fig. 2 as vertical pitch lines and horizontal thrust lines. The point and rate of transition can vary depending on the aircraft configuration and operating conditions. Fig. 3 is a representative gamma-V diagram for the CESTOL-type aircraft between the two regimes. Here the lines do not clearly follow the frontside or the backside convention. This highly nonlinear flight envelope for CESTOL aircraft is further complicated by control coupling between the engine and aerodynamic control effectors. Engine-aerodynamic coupling is caused by the engine location and advanced technology for CESTOL. Thus, the system complexity involving engine dynamic and aerodynamic coupling necessitates integrated flight-propulsion control for precision outer-loop control of the vehicle's velocity and flight path.

The adaptive implementation of the data-based predictive controller serves as an integrated flightpropulsion controller for the outer-loop control of STOL and provides optimal control inputs for the current system. Results in this paper show that the controller successfully tracks velocity while attempting to maintain flight path angle, using longitudinal command, thrust and flap setting as the control inputs.

\section{A. Simulation Results}

The data-based predictive controller described in this paper has been implemented on a full non-linear simulation of a CESTOL-type aircraft. ${ }^{12}$ A control diagram of the current implementation is shown in Fig. 4. This control architecture is a variant of the integrated flight-propulsion control design documented in Ref. 11. The Stability and Control Augmentation System is an adaptive dynamic inversion flight control system developed at NASA Ames Research Center. ${ }^{12}$ 
Input data for the data-based predictive controller is chosen to consist of inputs that impact the aircraft response and output data is chosen to consist of variables relevant to the system's dynamic state and controlled response. The recorded data types are selected carefully during the control design process to eliminate unnecessary inputs. Inputs that do not affect the system response add to the computation time of the controller. In general, the computation time is proportional to the square of the number of inputs. In this study, the information available to the data-based predictive controller is sensed velocity and flight path angle, and the controller maintains a record of its past command values for use in computation, which consist of thrust, flap and longitudinal commands.

For linear systems, as stated previously, $p$ is selected such that $m p \geq n+2 f+1$ and $0 \leq q \leq p$, where $n$ is the system order, $f$ is the number of distinct disturbance frequencies, and the 1 accounts for a constant disturbance if present. In this context, the exact number of states $n$, is unknown, so a large value of $p$ is used. The choice of $q$ primarily affects the aggressiveness of the controller, with smaller values of $q$ resulting in more aggressive controllers. The length $s$ of the prediction horizon is typically chosen to be as long as possible to improve the steady state performance and stability of the controller. As $s$ increases, the control solution approaches the solution for infinite-horizon optimal control. Since, for this application, only past data is used for the identification, the model being identified is $s$ time steps in the past, and therefore a large $s$ can degrade the accuracy of the controller gains if the dynamics of the system vary with time. Thus, a balance between stability and accuracy must be reached.

Weighting matrix $Q$ is chosen as a diagonal matrix, with values selected to reflect the relative magnitudes of the acceptable tracking errors. For this study, errors of 5 knots in velocity and 0.5 degrees in flight path angle are considered acceptable. The magnitudes of weighting matrices $R$ and $R_{S}$ relative to $Q$ affect the aggressiveness of the controller. Large weightings result in a less aggressive controller. The effects of $R$ and $R_{S}$ are not mutually exclusive, and must be balanced to achieve the desired results. For this implementation, $R$ and $R_{S}$ are also diagonal matrices.

The value of $S$ is constructed from a linear longitudinal aircraft model that was hand-tuned to include the effects of the flap. The gains $G, H$, and $K$ in $\gamma$ are updated every time step, with an initial covariance matrix $\Theta(0)=\mathbf{I} \cdot 10^{3}$. While the gains $\gamma$ can be adapted from zero, this strategy may have undesirable transients present during convergence of the parameters. For this application, an initial estimate of $\gamma$ is made by constructing $\mathbf{A}_{1}, \mathbf{A}_{2}$, and $M_{1}$ from Eq. (5), and the gains are then updated continuously during the simulation using the recursive least-squares estimation in Eq. (13) and Eq. (14). The control error of the estimation algorithm in Eq. (13) is subject to a dead-band when the L2 norm of the error vector is below a predetermined threshold. At the beginning of the simulation, random inputs are applied to excite the system and the gains are allowed to converge for a few seconds before the gains are used. The system behavior during this convergence window is not shown.

Simulation results in Fig. 5 show the performance of the controller tracking the commanded velocity. The commanded velocity is chosen to lead the aircraft through the transition from the frontside to the backside of the power curve. The high speed, low speed and middle speed correspond to the three flight conditions shown in Figs. 1-3, respectfully. The controller aircraft tracks the commanded velocity with an acceptable steady state error and well-damped transient behavior. The well-damped transient, which is desirable for passenger aircraft where comfort and slow accelerations are preferred, is a result of large control input and control smoothing weightings.

Fig. 6 shows the control inputs as percentage of total range for the longitudinal, flap, and thrust commands. For thrust, a value of zero corresponds to no thrust and a value of one corresponds to full thrust; for flap, a value of zero corresponds to no flap deflection and a value of one corresponds to full flap deflection; for longitudinal command, a value of negative one corresponds to a minimum pitch rate command and a value of one corresponds to a maximum pitch rate command. Both the commanded and achieved control inputs, which are subject to saturation and inner-loop augmentation, are presented in Fig. 6 . For the current simulation, longitudinal, flap, and thrust commands are rate and position limited. The flaps have a very slow response compared to the longitudinal command, and the engines have an even slower response. The plots of commanded and achieved thrust and commanded and achieved flap show that the controller successfully avoids rate saturation for these actuators. The longitudinal command is the command sent to the inner-loop adaptive stability and control augmentation system, which is a ratecommand attitude-hold controller. Thus, the small pulses in longitudinal command input corresponds to 
step commands in pitch that are achieved and maintained by the augmented longitudinal command. The pitch attitude response of the system is seen in Fig. 7.

As shown in Fig. 6, an increase in the commanded velocity is correlated with an increase in thrust command, which is consistent with the frontside control technique for controlling velocity. At the same time, an increase in the commanded velocity is correlated with a decrease longitudinal command that results in a decrease in pitch angle. This control input is consistent with the backside control technique for controlling velocity. An increase in the commanded velocity causes also corresponds to a decrease in the commanded flap, thus reducing the drag of the vehicle and consequently increasing the velocity. Similarly, a decrease in commanded velocity is correlated with a decrease in thrust command, increase in longitudinal command and decrease in commanded flap. The data-based predictive controller, therefore, controls the velocity using the frontside convention for the thrust command and the backside convention for the longitudinal command regardless of the flight condition of the simulated aircraft rather than employing a frontside technique at high speeds and transitioning to a backside technique as slow speeds.

Further investigation of the relationship between control parameters and the controlled systems is necessary to understand why the data-based predictive controller adopted the mixed control convention. One contribution may be the adaptive behavior of the controller gains, represented in Fig. 8, as the changes in selected gain values over time. Only significant estimation errors, which occur at the beginning of the simulation or when the vehicle accelerates and decelerates, are sufficient to cause updates to the control laws resulting in fixed controller gains during the later half of the simulation.

The performance of this controller in maintaining constant flight path angle is shown in Fig. 8. The flight path angle decreases when the velocity increases and increases as the velocity decreases, corresponding to a dive when the airplane accelerates and climbing when the aircraft decelerates. The magnitude of these deviations in flight path angle during acceleration and deceleration are unacceptable, but the flight path angle returns to an acceptable steady state error. The change in altitude resulting from deviations in flight path angle is shown in Fig. 9. The maximum change in altitude is around 500 feet over the 2000 second of simulation.

\section{Conclusions}

An adaptive formulation of data-based predictive control is presented and implemented as an integrated flight-propulsion controller for a CESTOL-type aircraft. The controller provides tracking of commanded velocity on both the front and backside of the power curve while attempting to maintain flight path angle. The data-based predictive controller is designed from input-output data without an intermediate system identification step, and the controller gains are adapted online. Results show that the controller learns to use a combination of backside and frontside techniques for both high and low speeds, rather than changing techniques according to speed. Further investigation of the relationship between control parameters and the controlled systems is necessary to understand why the data-based predictive controller adopted this mixed control convention, and to understand the deviation in flight path angle during acceleration and deceleration. 


\section{References}

${ }^{1}$ Richalet, J., Rault, A., Testud, J. L., and Papon, J., "Model Predictive Heuristic Control: Applications to Industrial Processes," Automatica, Vol. 14, No. 5, 1978, pp. 413-428.

${ }^{2}$ Clarke, D. W., Mohtadi, C., and Tuffs, P. S., "Generalized Predictive Control - Parts I and II," Automatica, Vol. 23, No. 2, 1987, pp. 137-148 and pp. 149-160.

${ }^{3}$ Clarke, D. W., and Mohtadi, C., "Properties of Generalized Predictive Control," Automatica, Vol. 25, No. 6, 1989, pp. 859-875.

${ }^{4}$ Darling, R. S. and Phan, M. Q., "Dynamic Output Feedback Predictive Controllers for Vibration Suppression and Periodic Disturbance Rejection," Proceedings of the $14^{\text {th }}$ AAS/AIAA Space Flight Mechanics Meeting, Maui, HI, February 2004.

${ }^{5}$ Darling, R. S. and Phan, M. Q., "Predictive Controllers for Simultaneous Tracking and Disturbance Rejection," Proceedings of the AIAA Conference on Guidance, Navigation, and Control, Providence, RI, August 2004.

${ }^{6}$ Barlow, J. S. and Phan, M. Q., "Model Predictive Control of Vehicle Formations," Proceedings of the AAS/AIAA Astrodynamics Specialist Conference, Mackinac Island, MI, August 2007.

${ }^{7}$ Barlow, J. S. and Phan, M. Q., "Adaptive Predictive Control of Vehicle Formations with Obstacle Avoidance," Proceedings of the AAS/AIAA Astrodynamics Specialist Conference, Mackinac Island, MI, August 2007.

${ }^{8}$ Barlow, J. S. and Phan, M. Q., "Direct Data-Based Model Predictive Controller Design," Proceedings of the AIAA Conference on Guidance, Navigation, and Control, Keystone, CO, August 2006.

${ }^{9}$ Goodwin, G.C. and Sin, K.S., Adaptive Filtering Prediction and Control, Prentice-Hall, Englewood Cliffs, NJ, 1984.

${ }^{10}$ Norton, B., "STOL Progenitors: The Technology Path to a Large STOL Aircraft and the C-17A", AIAA Case Study, American Institute of Aeronautics and Astronautics, Inc., Reston, Virginia, 2002.

${ }^{11}$ Franklin, J. A., Hynes, C. S., Hardy, G. H., Martin, J. L., Innis, R. C., "Flight Evaluation of Augmented Controls for Approach and Landing of Powered-Lift Aircraft," AIAA Journal of Guidance, Control and Dynamics, Volume 8, Number 5, September-October 1986, pp. 555-565.

12 Acosta, D. M., KrishnaKumar, K., Kaneshige, J. T., "Adaptive Control for ESTOL Design Abstractions-Performance Analysis," AIAA's 46th Aerospace Sciences Meeting and Exhibit, AIAA, Reno, Nevada, January 2008, AIAA-2008-0879 


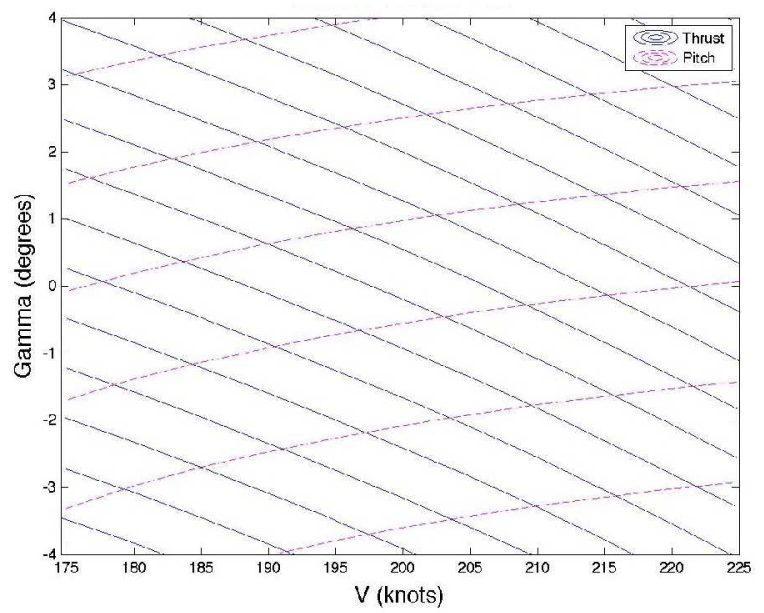

Figure 1 Gamma-V Plot: High speed indicating frontside technique

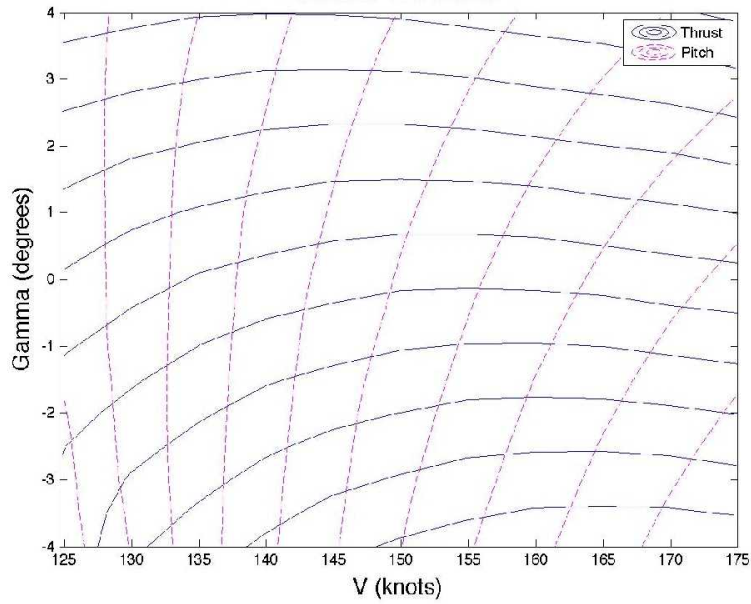

Figure 2. Gamma-V Plot: Low speed indicating backside technique

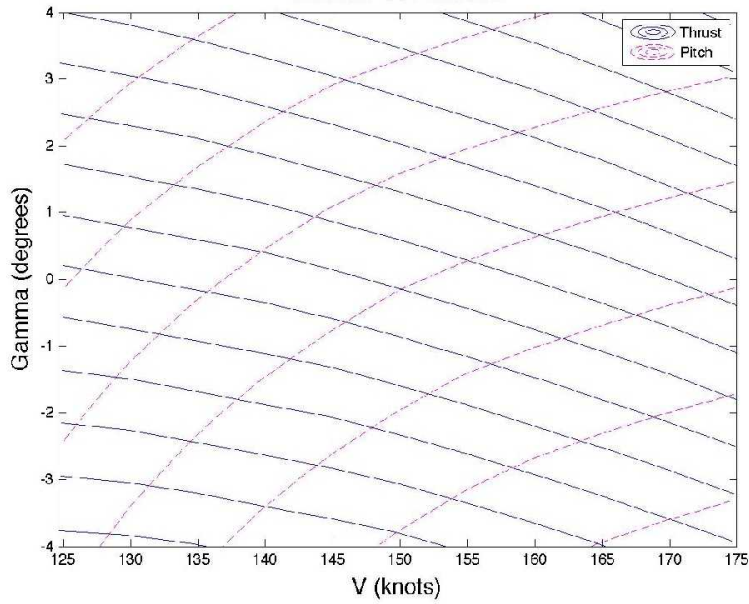

Figure 3. Gamma-V Plot: Medium speed indicating transition 


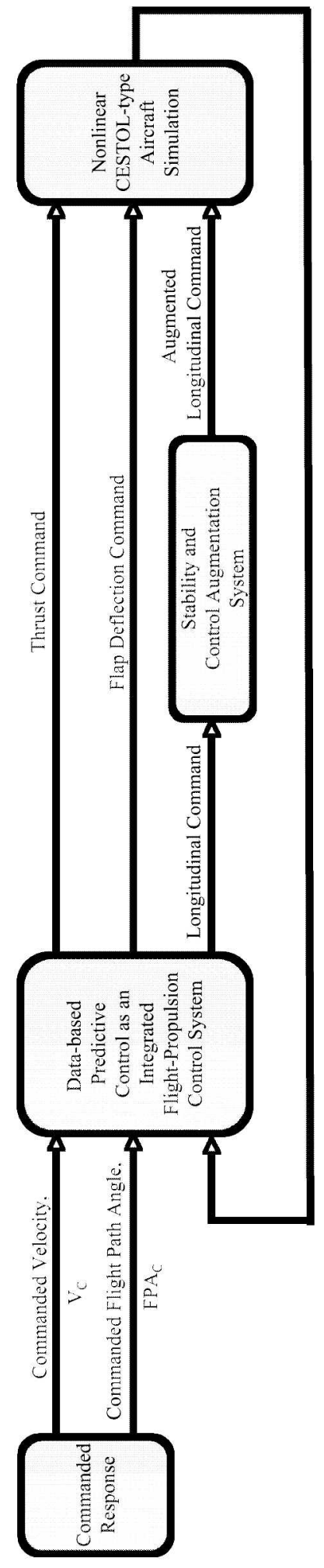

Figure 4. Control Diagram 


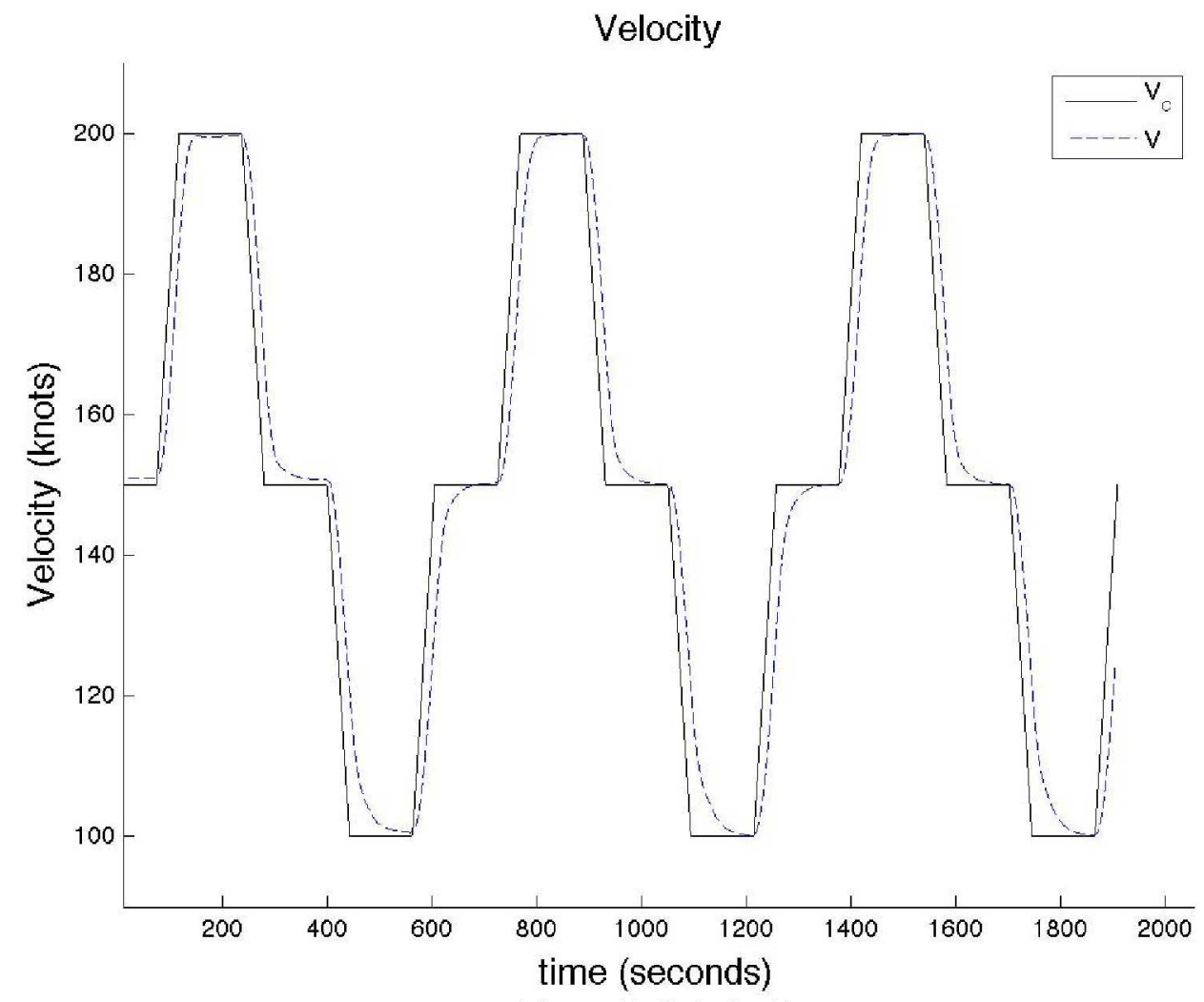

Figure 5. Velocity Response 


\section{Thrust Command}
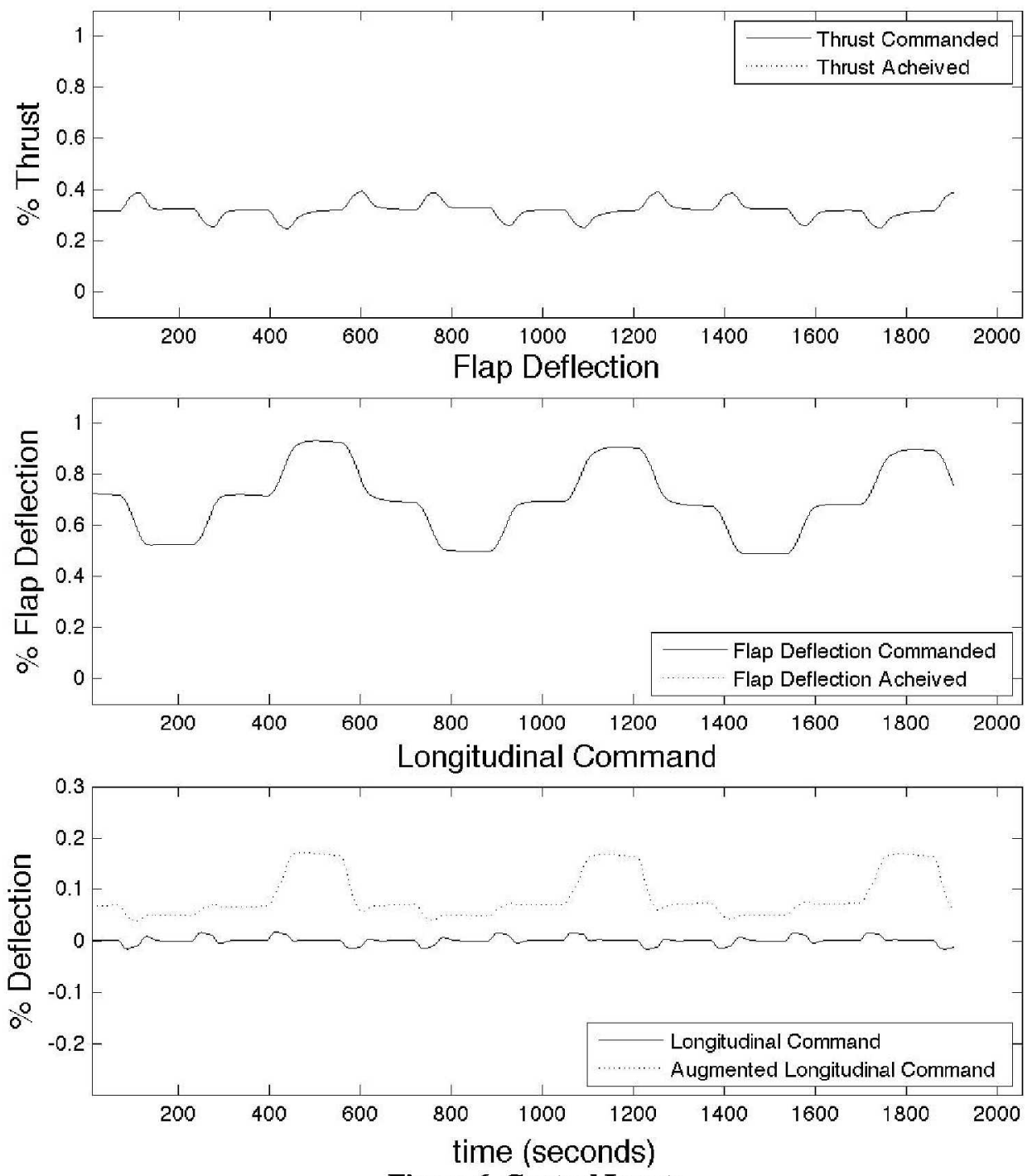

Figure 6. Control Inputs

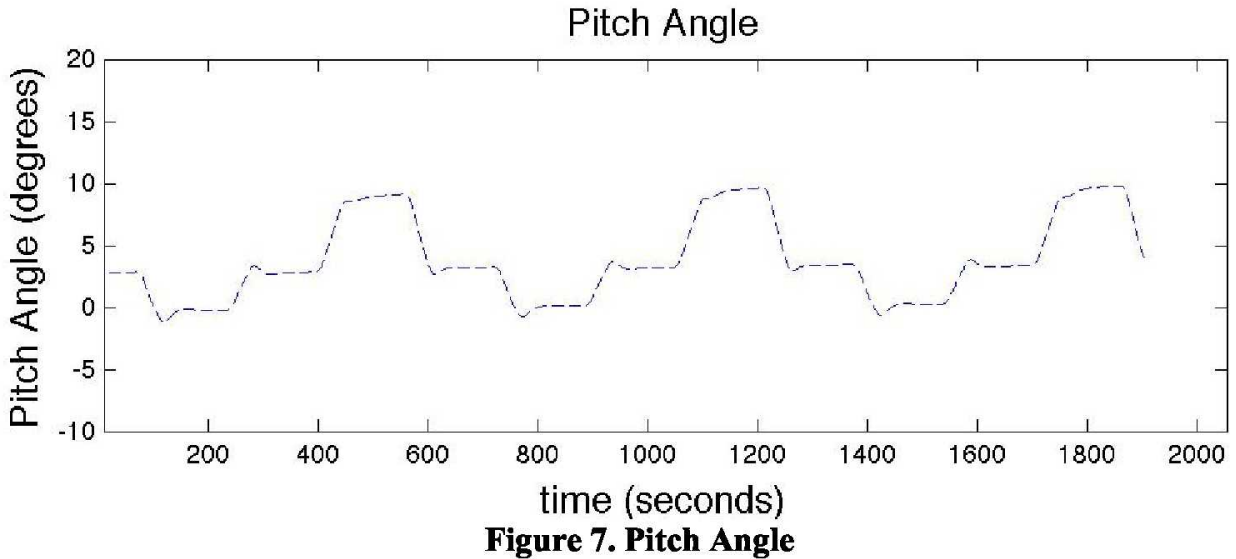




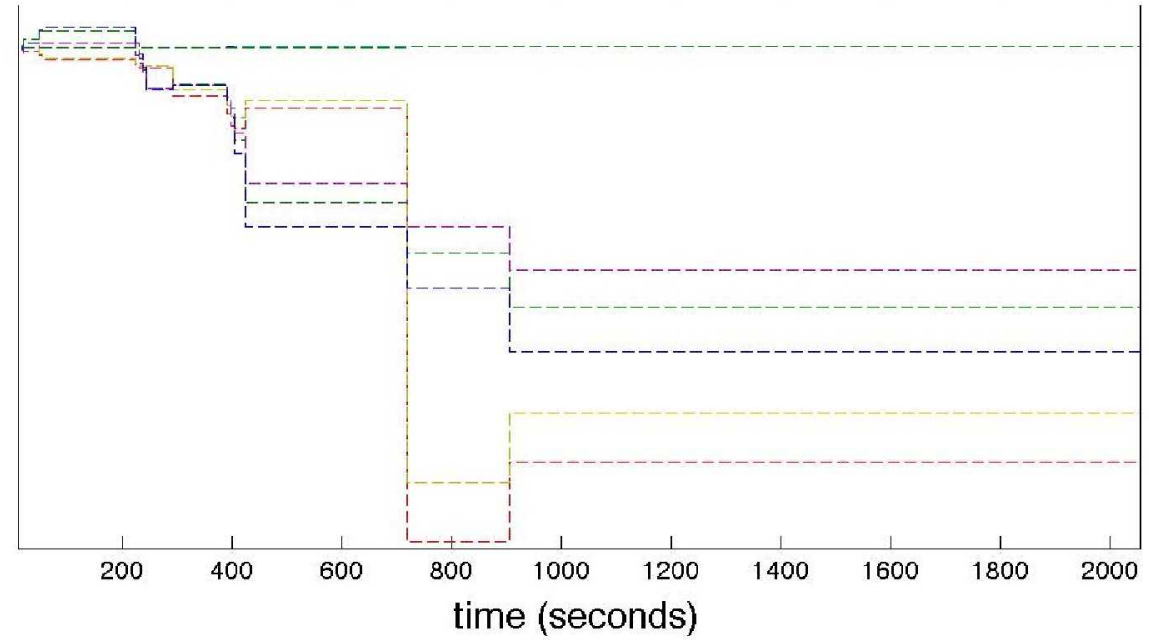

Figure 8. Convergence of controller gains

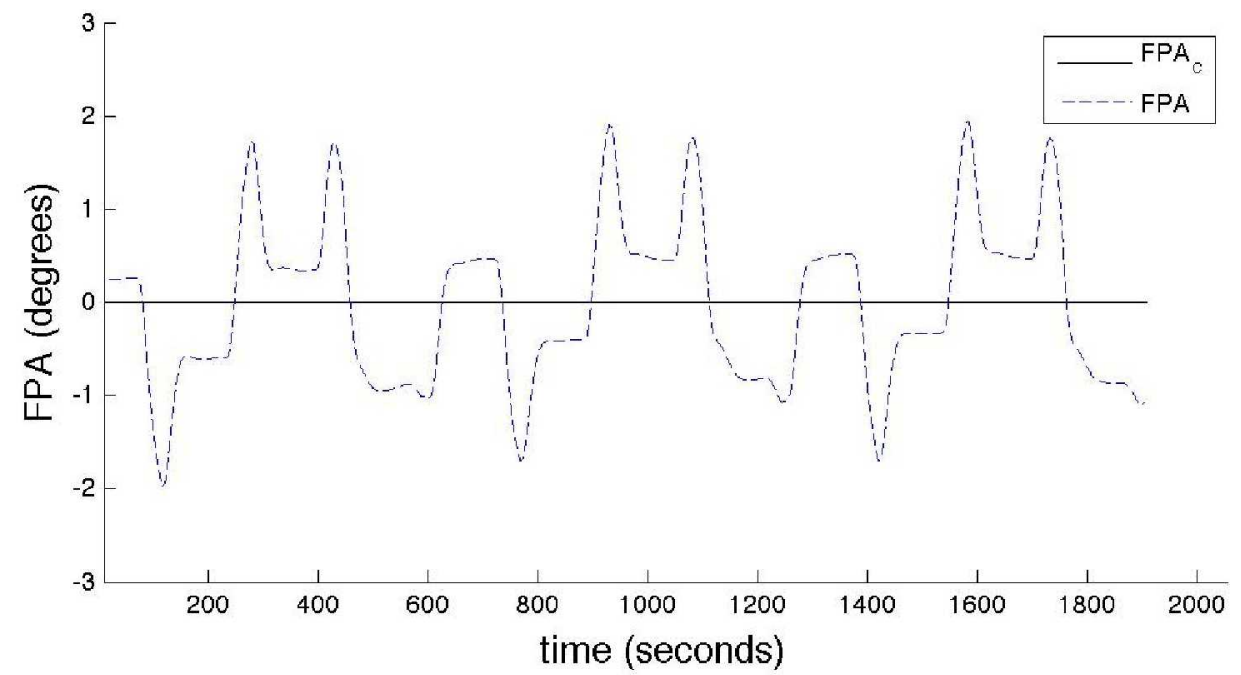

Figure 9. Flight Path Angle

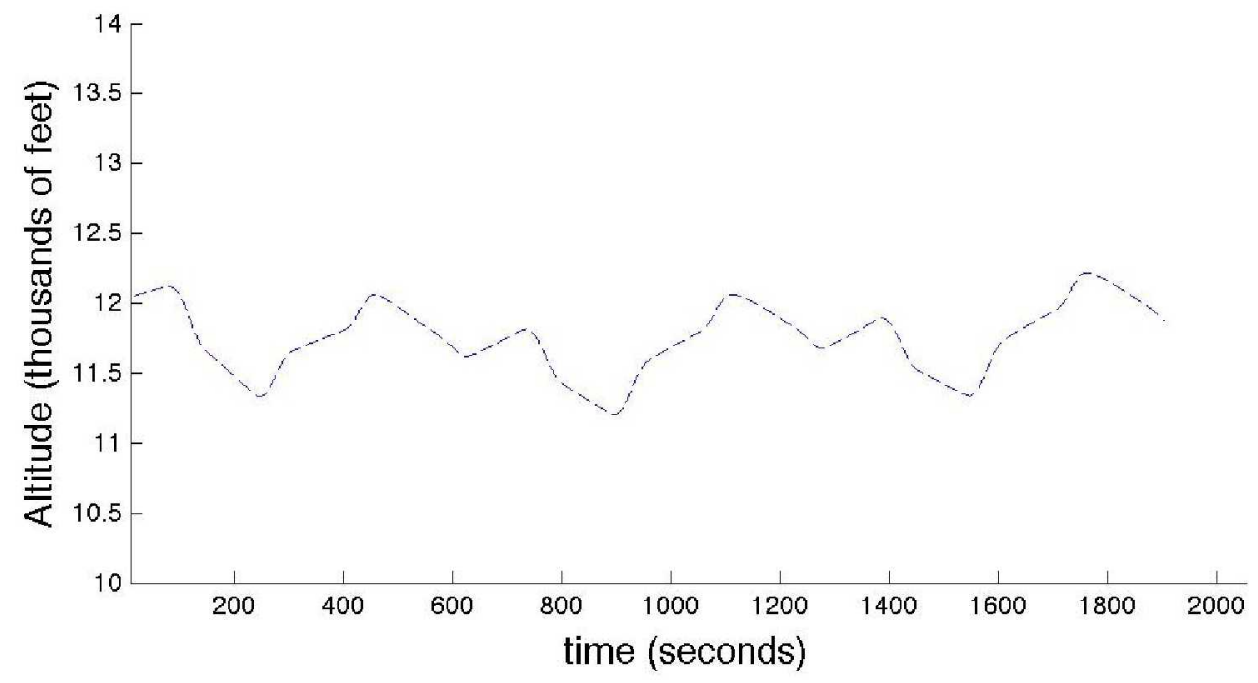

Figure 10. Altitude 